\title{
NEAR-RINGS OF MAPPINGS ON FINITE TOPOLOGICAL GROUPS
}

\author{
GORDON MASON \\ (Received September 7, 1982; revised September 5, 1983)
}

Communicated by J. H. Rubinstein

\begin{abstract}
When $G$ is a topological group, the set $N(G)$ of continuous self-maps of $G$, and the subset $N_{0}(G)$ of those which fix the identity of $G$, are near-rings. In this paper we examine the (left) ideal structure of these near-rings when $G$ is finite. $N_{0}(G)$ is shown to have exactly two maximal ideals, whose intersection is the radical. In the final section we investigate subnear-rings of $N_{0}(G)$ determined by certain continuous elements of the endomorphism near-ring.
\end{abstract}

1980 Mathematics subject classification (Amer. Math. Soc.): primary 16 A 76; secondary 22 A 99.

\section{Introduction}

Let $G$ be a topological group written additively. We denote by $N(G)$ the set of continuous maps $f: G \rightarrow G$, and by $N_{0}(G)$ those elements of $N(G)$ for which $f(0)=0$. When no confusion can arise, the notation will not indicate the topology used, but we will reserve $T(G)$ and $T_{0}(G)$ for the case when $G$ has the discrete topology. $N(G)$ and $N_{0}(G)$ are near-rings with identity under pointwise addition and composition of functions. For general results on near-rings, the reader is referred to Pilz [8] and in this paper all near-rings will be right near-rings. Unless otherwise stated $G$ will denote a finite group, and in the next section we will apply some ideas of Hofer [3] to obtain information about the (left) ideals in $N(G)$ and $N_{0}(G)$. In the third section we look at two subnear-rings of $N_{0}(G)$ determined by 
endomorphisms, namely the intersection of $N_{0}(G)$ with the endomorphism nearring $E(G)$, and the near-ring distributively generated by continuous elements of $E(G)$. In particular, the orders of these near-rings are obtained for classes of near-rings for which the order of $E(G)$ is known (see [1], [5], [6], [7]).

\section{Ideals in $N(G)$ and $N_{0}(G)$}

If $G$ is a finite topological group, the topology is determined by a normal subgroup. That is, $G$ has a topology if and only if $H$ is a normal subgroup such that a basis for the open sets of $G$ consists of the cosets of $H$ (see for example [2]). Then $G$ is disconnected, the connected component of 0 being $H$. Now, if $G$ is an infinite Hausdorff group and $C$ is the connected component of 0 , Hofer [3] defined $M_{0}=\left\{f \in N_{0}(G) \mid f^{-1}(0)\right.$ contains a clopen set about 0$\}, P=P(C)=\{f$ $\in N(G)$ range of $f \subseteq C$ \} and $P_{0}=P \cap N_{0}$ and observed that $P$ is an ideal in $N(G)$ and $P_{0}$ and $M_{0}$ are ideals in $N_{0}(G)$ such that $M_{0} \backslash P_{0} \neq \varnothing$. In our case, although $G$ is not $T_{2}$, these results are still true. Moreover, $H$ is the smallest clopen set about 0 so $M_{0}=\{f \mid f(H)=0\}$ which is sometimes written $(0: H)$ or Ann $H$. In fact, it is known that every left ideal of $T_{0}(G)$ is of the form Ann $S$ for some $S \subset G$ ([8] Corollary 7.28), and that these all intersect down to left ideals in $N_{0}(G)$. We observe that $M_{0}$ is one of these intersections and is, in fact, an ideal, although $T_{0}(G)$ has no ideals.

THEOREM 2.1. If $H$ is a subgroup of index 2 in a group $G$ of order $2 n$ then $|N(G)|=4 \cdot n^{2 n},\left|N_{0}(G)\right|=2 \cdot n^{2 n-1},|P|=n^{2 n},\left|P_{0}\right|=n^{2 n-1}$ and $\left|M_{0}\right|=2 \cdot n^{n}$ where, as above, $P=\{f \in N \mid f(G) \subseteq H\}$ and $P_{0}=P \cap N_{0}$.

Proof. The only non-trivial open sets are $H$ and $g+H(g \notin H)$. Therefore, $f$ : $G \rightarrow G$ is continuous if and only if it is one of the following: (a) $f^{-1}(H)=H$ and $f^{-1}(g+H)=g+H$, (b) $f^{-1}(H)=g+H$ and $f^{-1}(g+H)=H$, (c) $f^{-1}(H)=$ $\varnothing$ and $f^{-1}(g+H)=G$, (d) $f^{-1}(H)=G$ and $f^{-1}(g+H)=\varnothing$. There are $n^{2 n}$ maps in each case. In $N_{0}(G)$ since $f^{-1}(0) \supseteq(0)$ only maps from (a) and (d) are allowed and there are now $n^{2 n-1}$ choices in each case. Clearly $\left|M_{0}\right|=2 \cdot n^{n}$. As for $P$ and $P_{0}$, more generally if $|H|=k$ and $|G|=m$, then any map $f: G \rightarrow G$ whose range is in $H$ is continuous so $|P|=k^{m}$, and it is easy to see that $\left|P_{0}\right|=k^{m-1}$.

As a corollary note that for $H$ of index $2, P_{0}$ is maximal in $N_{0}$ being also of index 2 . 
Now Hofer has shown that when $G$ is $T_{2}, P(C)$ is often the unique maximal ideal in $N(G)$, for example when $|G / C|=n>2$. Actually the proof in that case is independent of the $T_{2}$ condition. It uses the canonical map $\psi: N(G) \rightarrow N(G / C)$ given by $\psi(f)(x+c)=f(x)+C$ whose kernel is $P(C)$ and which is onto when $C$ is open. Now the isomorphism $N(G / C) \simeq N(G) / P$ induces $N_{0}(G / C) \simeq$ $(N(G) / P)_{0}$ but the latter is not a priori isomorphic to $N_{0}(G) / P_{0}$. In other words a near-ring surjection $N \rightarrow R$ induces a map $N_{0} \rightarrow R_{0}$ which may not be onto. However, examining $\psi$ more closely, let $\nu: G \rightarrow G / C$ be the canonical map and $k$ : $G / C \rightarrow G$ a map for which $\nu k$ is the identity. Then for every $g \in N(G / C)$, $g=\psi(f)$ where $f(x)=k(g(x+C))$. In our case $C=H$ is open, $G / H$ is finite and so discrete and $\psi$ restricted to $N_{0}(G)$ produces a near-ring homomorphism with kernel $P_{0}$. Moreover, we can choose $k$ so that $k(H)=0$ so the preimage $f$ of $g \in N_{0}(G / C)$ is actually in $N_{0}(G)$ as required. We have

THEOREM 2.2. $P_{0}$ is a maximal ideal in $N_{0}(G)$.

Clearly, however, $N_{0}$ does not have a unique maximal ideal since $M_{0}$ is contained in some maximal ideal(s) and $M_{0} \not \subset P_{0}$. We shall see below that for $G$ finite, $M_{0}$ is maximal and that $P_{0}$ and $M_{0}$ are the only maximal ideals. For now we simply observe (even for $G$ infinite) that Zorn's lemma applies to $S=$ (ideals $\left.I \mid I \backslash P_{0} \neq 0\right\}$ and the maximal elements so obtained must be maximal ideals.

In Lemma 2.12 of [3] it was proved that if $G$ is $T_{2}$ and $I$ is an ideal of $N_{0}(G)$ such that $I \backslash P_{0} \neq \varnothing$ then $I$ contains all functions whose range is finite. The conclusion is false for general non-Hausdorff groups; for example when $G$ is finite this would say $I$ is all of $N_{0}(G)$, but we know $M_{0}$ is a proper ideal satisfying $M_{0} \backslash P_{0} \neq \varnothing$. However, the following weaker statement is true, and in fact it is a valid replacement for Lemma 2.12 in [3, Theorem 3.3 and Theorem 3.8(b)] (see next Corollary). Let $R(f)$ denote the range of $f$ and call its order the rank of $f$.

Proposition 2.3. Let $G$ be any disconnected group and $C$ the connected component of 0 . ( $G$ need not be Hausdorff or finite.)

(a) If $I$ is an ideal in $N_{0}(G)$ such that $I \backslash P_{0} \neq \varnothing$, then $I$ contains all functions $f$ with $R(f)=\{0, a\}$ where $a \notin C$. Moreover, $I$ does not contain all functions of rank 2 , in the case $G$ is finite.

(b) If $|G / C|>2$ and $I$ is an ideal in $N(G)$ with $I \backslash P \neq \varnothing$ then $I$ contains all functions $f$ with $R(f)=\{c, a\}$ where $c \in C, a \notin C$.

Proof. The proof given in [3, Lemma 2.12] remains valid except at one point. It is noted that when $G$ is $T_{2}$, if $R(g)=\{0, a\}$ then $g^{-1}(0)$ and $g^{-1}(a)$ are clopen. For arbitrary $G$, this will be true if $a \notin C$. The result follows. 
Suppose $I$ contains all functions of rank 2. We show $I$ contains $P_{0}$ which is a contradiction. Proceeding by induction let $R(f)=\left\{0, h_{2}, \ldots, h_{n}\right\} \subset H$. Put $f_{1}(x)$ $=0$ if $f(x)=0, f_{1}(x)=h_{2}$ otherwise. Then $f_{1}$ is continuous and by hypothesis it is in $I$. Also by induction $f-f_{1} \in I$ so $f \in I$.

Corollary. For $G$ as in the proposition, if $|G / C|=n>2$ then $P(C)$ is the unique maximal ideal of $N(G)$ (see [3, Theorem 3.8b]).

We now show $M_{0}$ is maximal, first recording the following characterization of continuity.

Lemma 2.4. If $\psi \in T_{0}(G)$ then $\psi \in N_{0}(G)$ if and only if $\psi(g) \in x+H \Rightarrow$ $\psi(g+H) \subseteq x+H$.

Proof. This is simply the observation that $\psi$ is continuous if and only if the inverse images of basic open sets (cosets) are open.

THEOREM 2.5. There is a near-ring isomorphism between $T_{0}(H)$ and $N_{0}(G) / M_{0}$.

Proof. Map $\psi: N_{0}(G) \rightarrow T_{0}(H)$ by restriction, noting that by the lemma $\left.\psi\right|_{H}$ is in $T_{0}(H)$. This is easily seen to be a near-ring homomorphism. Moreover, it is onto since any $f \in T_{0}(H)$ can be extended to a continuous map on $G$ by, for example, setting $f(x)=0$ for all $x \notin H$. Finally, $\operatorname{ker} \psi=\left\{f|f|_{H}=0\right\}=M_{0}$.

Since by [8, Theorem 7.30] $T_{0}(H)$ is simple we have

CoRollary. $M_{0}$ is a maximal ideal in $N_{0}$.

We refer the reader to [8, Chapter 5] for definitions of the radicals.

THEOREM 2.6. All the radicals $J_{i}$ of $N_{0}(G)$ coincide and equal $M_{0} \cap P_{0}$.

Proof. $J=M_{0} \cap P_{0}$ is nilpotent since $f \in J$ implies $f(H)=0$ and $f(G) \subset H$. Therefore for all $f, f_{1} \in J f f_{1}(G) \subset f(H)=0$. Hence $J \subseteq J_{1}$. On the other hand by [8, Theorem 5.42] $J_{1}=J_{2}=\cap$ all maximal ideals $\subseteq J$. Hence $J=J_{1}$ and by [8, Theorem 5.48] $J=J_{0}=J_{1 / 2}$ also.

TheOREM 2.7. $M_{0}$ and $P_{0}$ are the only maximal ideals of $N_{0}$. 
Proof. Suppose $I$ is maximal, $I \neq P_{0}$. We show $I \supseteq M_{0}$. By the previous Theorem $I \supset M_{0} \cap P_{0}$ so we show $I$ contains all $f$ with $f(H)=0$ and $R(f) \not \subset H$. Proceeding by induction, it is true for rank $f=2$ by Proposition 2.3. Suppose $f \in M_{0}$ has rank $n, R(f) \not \subset H$, that is, $R(f)=\left\{0, a_{2}, \ldots, a_{n}\right\}$ with $a_{2} \notin H$. Define $f_{1}(x)=0$ if $x \in f^{-1}(H), f_{1}(x)=a_{2}$ otherwise. Then $f_{1}$ is continuous, $\operatorname{rank}\left(f-f_{1}\right)=n-1$ and $f-f_{1} \in I$ either because $R\left(f-f_{1}\right) \subset H$ or by induction. Also $f_{1} \in I$ by Proposition 2.3 so $f \in I$ as required.

To exploit Theorem 2.5 further we note as mentioned earlier that for any finite group $G$ the left ideals in $T_{0}(G)$ are precisely of the form Ann $S$ for $S \subset G$, so maximal ones are obtained for $S=\{g\}$ and minimal ones for $S=G-\{g\}$. Following Pilz [8] we will denote the latter by $L_{g}$ and put $\overline{L_{g}}=L_{g} \cap N_{0}$. By [8, 7.18] we have $L_{g}=T_{0}(G) e_{g}$ where $e_{g}$ is the idempotent given by $e_{g}(g)=g$, $e_{g}(x)=0$ for all $x \neq g$. Note that $G$ is an $N(G)$-group and an $N_{0}(G)$-group under canonical action.

LEMMA 2.8. ${ }_{N(G)} G$ is strongly monogenic and ${ }_{N_{0}(G)} G$ is monogenic.

Proof. All constant maps are in $N(G)$ so $N(G) \cdot g=G$ for every $g$. In the case of $N_{0}(G)$ if $g \notin H$ then for all $x$ there is $f \in N_{0}$ with $f(g)=x$ so for those $g$, $N_{0}(G) \cdot g=G$.

THEOREM 2.9. (a) $e_{g}$ is continuous if and only if $g \in H$ and then $\overline{L_{g}}=N_{0}(G) e_{g}$.

(b) $\overline{L_{h}} \simeq N_{0}(G) /$ Ann $h$ for all $h \in H$ and $\overline{L_{g}} \simeq H$ for all $g$.

(c) $G \simeq N(G) /$ Ann $g$ (for all $g \in G$ ) as $N(G)$-groups. If $g \notin H \quad G \simeq$ $N_{0}(G) /$ Ann $g$ as $N_{0}(G)$-groups.

(d) $\bar{L}_{g}$ is a minimal $N_{0}(G)$-subgroup of $N_{0}$ for all $g$ and Ann $h$ is a maximal $N_{0}(G)$-subgroup for all $h \in H$. (So they are respectively minimal and maximal left ideals also.)

Proof. (a) Clearly $e_{h}$ is continuous if $h \in H$ and on the other hand if $g \notin H$, $e_{g}^{-1}(H)=G \backslash\{g\}$ is not open. Moreover, $\overline{L_{g}}=L_{g} \cap N_{0}=T_{0}(G) e_{g} \cap N_{0}(G)=$ $N_{0}(G) e_{g}$.

(b) Map $\alpha: N_{0}(G) \rightarrow \overline{L_{h}}=N_{0}(G) e_{h}$ by $\alpha(f)=f e_{h}$. This is an $N_{0}(G)$-epimorphism whose kernel is Ann $h$. Now let $\beta: H \rightarrow \overline{L_{g}}$ be given by $\beta\left(h_{0}\right)=f$ where $f(g)=h_{0}, f(x)=0$ for all $x \neq g$. Then $\beta$ is an $N_{0}(G)$-homomorphism which is $1-1$. It is also onto for if $f \in \bar{L}_{g} f(x)=0$ except when $x=g$ and then $f(g)=a$, where by continuity $a \in H$. Thus $f=\beta(a)$ as required.

(c) By Lemma 2.8 and [8, Proposition 3.4] we have $G \simeq N(G) /$ Ann $g$ for all $g$ and $G \simeq N_{0}(G) /$ Ann $g$ for all $g \notin H$.

(d) We show $\overline{L_{h}}$ is minimal by showing for all $0 \neq f \in \overline{L_{h}} N_{0}(G) f=\overline{L_{h}}$. Now $f(x)=0 \forall x \neq h$ and $f(h) \neq 0$. Define $g$ by $g(f(h))=h, g=0$ otherwise. Then 
$\underline{g} \in N_{0}$ and $e_{h}=g f \subset N_{0} f$. Hence $\overline{L_{h}}=N_{0}(G) e_{h} \subset N_{0} f$ as required. Clearly $\overline{L_{g}} \simeq \overline{L_{h}}$ for all $g$ so that $\bar{L}_{g}$ are minimal also. Applying (b) we have Ann $h$ is a maximal $N_{0}$-subgroup for all $h \in H$.

Having seen the Ann $h$ are maximal $N_{0}$-subgroups we turn our attention to Ann $g, g \notin H$.

LEMMA 2.10. The only $N_{0}$-subgroup of $G$ is $H$.

Proof. If $K$ is an $N_{0}$-subgroup of $G$, it is a subgroup of $(G,+)$ such that $\psi(K) \subseteq K$ for all $\psi$ in $N_{0}$. This is certainly true of $H$. On the other hand, if $H \backslash K \neq \varnothing$ let $h \in H \backslash K, h \neq 0$ and define $\psi$ by $\psi(g)=h$ for all $g \neq 0$, and $\psi(0)=0$. Then $\psi$ is continuous but $\psi(K) \nsubseteq K$. Finally if $H \varsubsetneqq K, K=\cup_{S}(g+H)$ for some set $S$ of coset representatives. There exists $g \in S \backslash H$ so define $\psi_{1}$ by $\psi_{1}(g+H)=y+H$ for any $y \notin S$, and $\psi_{1}$ the identity on the rest of $G$. Then again $\psi_{1}$ is continuous but $\psi_{1}(K) \not \subset K$.

Put $S_{g}=\left\{f \in N_{0} \mid f(g+H) \subseteq H\right\}$ for $g \notin H$.

Proposition 2.11. $S_{g}$ is a maximal left ideal and maximal $N_{0}$-subgroup properly containing Ann $x$ for all $x \in g+H$. Moreover $S_{g} \simeq S_{x}$ for all $x, g \notin H$ and $\bigcap_{g \notin H} S_{g}=P_{0}$.

Proof. $S_{g}$ is a normal subgroup of $\left(N_{0}(G),+\right)$ since $H$ is normal in $G$. Moreover for all $\psi, \alpha \in N_{0}$ and $f \in S_{g}$ let $x=[\psi(\alpha+f)-\psi \alpha](g+h)=$ $\psi\left(\alpha(g+h)-h_{1}\right)-\psi \alpha(g+h)$ where $f(g+h)=h_{1} \in H$. By Lemma 2.4, $x \in$ $H$, so $S_{g}$ is a left ideal. By Theorem 2.8(c) $G \simeq N_{0}(G) /$ Ann $g$ and under this isomorphism (which comes from the evaluation map) $S_{g}$ corresponds to $H$. Thus the $S_{g}$ are all isomorphic (in fact $S_{g}=S_{x}$ if $x \in g+H$ ). By Lemma 2.9, the only $N_{0}(G)$-subgroup of $G$ is $H$ so the $S_{g}$ are the only $N_{0}(G)$-subgroups of $N_{0}$ which contain Ann $g$. Finally $P_{0} \subset S_{g}$ for all $g$ and if $f \in \cap S_{g}$ then $f(g+H) \subseteq H$ for all $g$, that is, $f \in P_{0}$.

\section{Subnear-rings of $N_{0}(G)$}

In this section, $G$ is again a finite topological group with topology determined by a normal subgroup $H$ and we will write $N_{0}(G)$ as $N_{H}$. Let $I, A$ and $E$ be the near-rings distributively generated by $\operatorname{Inn} G$, Aut $G$ and End $G$ which are respectively the groups of inner automorphisms, automorphisms and endomorphisms of 
$G$. There is an extensive literature on these near-rings for various classes of finite groups (see for example [1], [4], [5], [6], [7]) and using these results we will examine two kinds of subnear-rings of $N_{H}$. The first is $E_{H}=N_{H} \cap E$, the near-ring of continuous maps in $E$, and the second is $C_{H}$, the near-ring distributively generated by the continuous elements in End $G$. ( $E_{H}$, although a subnearring of $E$, is not necessarily distributively generated.) Since every inner automorphism is continuous in all topologies we have for all $H$

$$
I \subseteq C_{H} \subseteq E_{H} \subseteq E .
$$

We shall see later that for $G=D_{8}$, the dihedral group of order 8, we have in fact a chain of maximal proper inclusions $I \varsubsetneqq A \varsubsetneqq C_{H} \subsetneq E_{H} \varsubsetneqq E$. At the other extreme, it may happen that $I=E$ (for example $G$ dihedral of order $2 n, n$ odd ([6]) or $G=S_{n}$ the symmetric group, for $n \geqslant 5$ ([1])). In such a case $E \subset N_{H}$ and the same is true whenever $H$ is fully invariant, in view of the next result.

Lemma 3.1. If $\psi \in$ End $G, \psi \in E_{H}$ if and only if $\psi(H) \subseteq H$. Also if $\psi \in$ Aut $G$, $\psi \in E_{H}$ if and only if $\psi H=H$.

Proof. Apply Lemma 2.4.

Thus for characteristic subgroups $H$ the sequence (1) can be modified to include $I \subseteq A \subseteq C_{H}$. To complete the example for $S_{n}, n=3$ or 4 , in each case the only normal subgroups are members of the derived series [9, page 112]) and these are fully invariant so again $E \subset N_{H}$.

Proposition 3.2. $J_{i}\left(N_{H}\right) \cap E \subseteq J_{i}(E)$ for all radicals $J_{i}$.

Proof. From [4] we know all radicals of $E$ coincide. Since $J\left(N_{H}\right)$ is nilpotent so is $J\left(N_{H}\right) \cap E$ and the result follows.

From the remarks following Theorem 16 in [4] we find that if $G$ has a unique fully invariant subgroup $H$ then $J(E)$ is precisely (in our notation) $M_{0} \cap P_{0} \cap E$. Thus in this case, equality holds in Proposition 3.2. (An example will be given later where equality does not hold.) To complete our discussion of $S_{n}, n \geqslant 5$, it is mentioned in [1] that $E\left(S_{n}\right)$ is close to being all of $T_{0}\left(S_{n}\right)$. We know $E \subseteq N_{H} \subseteq T_{0}$.

Proposition 3.3. $E\left(S_{n}\right)=N_{H}\left(S_{n}\right)$ when $H=A_{n}$.

Proof. From [1] $E=N+\left(T_{0}(H) \oplus Z_{2}\right)$ is a semi-direct sum where $N=M_{0}$ $\cap P_{0}$ (again in our notation). Moreover $T_{0}(H)$ is a direct sum of $n ! / 2$ subgroups, each isomorphic to $H$. Thus $|E|=\left|N_{H}\right|$ using Theorem 2.1. 
To obtain information about $C_{H}$ and $E_{H}$ we use the following decomposition procedure from, for example, [7]. If $R$ is a d.g. near-ring, one decomposes the generators $r_{i}$ by an idempotent $e_{1}$ to obtain elements of the form $r_{i}-e_{1} r_{i}$, and of the form $e_{1} r_{i}$, the latter generating a group $M_{1}$. The elements of the first form are conjugated by elements of $M_{1}$ and these conjugates generate $A_{1}$. Choose a second idempotent $e_{2} \in A_{1}$ and again form the conjugates of all $x-e_{2} x\left(x \in A_{1}\right)$ by elements of the group generated by the $e_{2} x$. These conjugates generate $D$ and $R=D+A_{1}+M_{1}$. The procedure may be iterated.

In particular if $G=D_{2 n}$ is the dihedral group of order $2 n$ for $n$ even with presentation $G=\left\langle a, b \mid a^{n}=b^{2}=a b a b=e\right\rangle$, following [7] we denote an endomorphism $\psi$ by $[s, t]$ where $\psi(a)=s$ and $\psi(b)=t$, and denote any map by the images of $\left(e, a, a^{2}, \ldots, a^{n-1} \mid b, a b, \ldots, a^{n-1} b\right)$ in that order. The endomorphisms are of six types:

(1) $\left[a^{y}, a^{x} b\right], \quad 0 \leqslant y, x \leqslant n-1$,

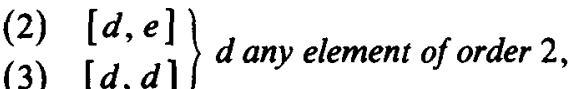

(4) $\left[e, a^{n / 2}\right]$,

$\left.\begin{array}{ll}\text { (5) }\left[a^{x} b, a^{x+n / 2} b\right] \\ \text { (6) }\left[a^{x} b, a^{n / 2}\right]\end{array}\right\} 0 \leqslant x \leqslant n-1$.

Let $H=\langle a\rangle$ be the cyclic normal subgroup of index 2. We now restrict to $n=4$ for ease of calculation. Then ([7]) $E=D+A_{1}+M_{1}$ where $D=$ $\left\{\left(e, e, a^{2}, a^{2} \mid e, e, a^{2}, a^{2}\right) \oplus\left(e, a^{2}, a^{2}, e \mid e, e, a^{2}, a^{2}\right)\right\}, A_{1}=\{(e, g$, $e, g \mid e, g, e, g) \mid g \in G\}$ and $M_{1}=\{(e, e, e, e \mid g, g, g, g) \mid g \in G\}$. Using Lemma 3.1 we see all 8 elements of $M_{1}$, all 4 elements of $D$ and the 4 elements of $A_{1}$ for which $g \in H$, are continuous. Thus $\left|E_{H}\right| \geqslant 128$. But since $|E|=256$ and there are endomorphisms which are not continuous, $\left|E_{H}\right|=128$. If on the other hand we topologize $G$ by $K=\left\{e, b, a^{2}, a^{2} b\right\}$ then there are 32 maps in $E_{K}$ which are a sum of continuous maps in each of $D, A_{1}$ and $M_{1}$ so $\left|E_{K}\right| \geqslant 32$. As we shall see later however the order of $E_{K}$ is actually 128.

Now we can obtain $C_{H}$ by applying the procedure outlined above. First, there are 29 continuous endomorphisms, namely all those from (1), those from (2) and (3) with $d=a^{2},(4)$, and the identity $[e, e]$. Using the idempotent $\gamma_{1}=[e, b]$ we get one form for $\alpha-\gamma_{1} \alpha$, namely $\beta=\left(e, a^{y}, a^{2 y}, a^{3 y} \mid e, a^{y}, a^{2 y}, a^{3 y}\right)$ for $0 \leqslant y$ $\leqslant 3$. The elements $\gamma_{1} \alpha$ are $\left[e, a^{x} b\right], 0 \leqslant x \leqslant 3,[e, e]$, and $\left[e, a^{2}\right]$ which generate $M_{1}=\{(e, e, e, e \mid g, g, g, g)\}$ as before. Conjugating $\beta$ by the $\gamma_{1} \alpha$ gives $\beta$ and $\beta_{1}=\left(e, a^{y}, a^{2 y}, a^{3 y} \mid e, a^{-y}, a^{-2 y}, a^{-3 y}\right)$. Choose the idempotent $\gamma_{2}=$ $\left(e, a, a^{2}, a^{3} \mid e, a, a^{2}, a^{3}\right)$ to get the single form $\gamma_{2} \beta=\gamma_{2} \beta_{1}=\beta$ so $A_{1}$ has 4 elements. Then $\beta-\gamma_{2} \beta=[e, e]$ and $\beta_{1}-\gamma_{2} \beta_{1}=\left(e, e, e, e \mid e, a^{2 y}, e, a^{2 y}\right)$ so 
conjugating these by $\gamma_{2} \beta=\beta$ gives only $\beta_{1}-\gamma_{2} \beta_{1}$ again and hence $D$ has 2 elements. Thus $\left|C_{H}\right|=64$.

TheOREM 3.4. If $n$ is even and $H=\langle a\rangle,\left|C_{H}\left(D_{2 n}\right)\right|=n^{3}$. Moreover for $n=4$, $I \varsubsetneqq A \varsubsetneqq C_{H} \varsubsetneqq E_{H} \varsubsetneqq E$ where the order of each near-ring is twice the preceding one.

Proof. For general $D_{2 n}$ the procedure outlined above can be generalized, giving $C_{H}=D+A_{1}+M_{1}$ where $|M|=2 n,\left|A_{1}\right|=n$ and $|D|=n / 2$. The second statement comes from the above discussion and [7].

Remark. The elements of $C_{H}$ are of the form $\sum \pm f_{i}$ where the $f_{i}$ are the continuous endomorphisms. In general, all possible ordered sums must be calculated. As an interesting consequence of the computer programme we used to exhibit the elements of $C_{H}\left(D_{8}\right)$ we found that (i) of the 20 continuous endomorphisms, only the 8 automorphisms plus the endomorphism $(e, e, e, e \mid b, b, b, b)$ were needed, (ii) only elements $\sum f_{i}$ (all + ) were needed, and (iii) all elements could be obtained from one particular ordering of the 9 generators. Also in producing the 32 elements of $A\left(D_{8}\right)$, (ii) and (iii) were true.

To complete the discussion of $D_{8}$, let $K=\left\{e, a^{2}, b, a^{2} b\right\}$. Then there are 24 continuous endomorphisms and the standard decomposition using the idempotents $\gamma_{1}=[e, b]$ and $\gamma_{2}=(e, a b, e, a b \mid e, a b, e, a b)$ show that $\left|C_{K}\right|=128$. Since there are endomorphisms which are not continuous, $\left|E_{K}\right|=128$ too. The only other normal subgroups are (i) $K_{1}=\left\{e, a b, a^{2}, a^{3} b\right\}$ and (ii) the centre $Z=$ $\left\{e, a^{2}\right\}$. By symmetry $C_{K_{1}} \cong C_{K}$, and every endomorphism is $Z$-continuous.

THEOREM 3.5. The continuous subnear-ring structure of $T_{0}\left(D_{8}\right)$ is given by

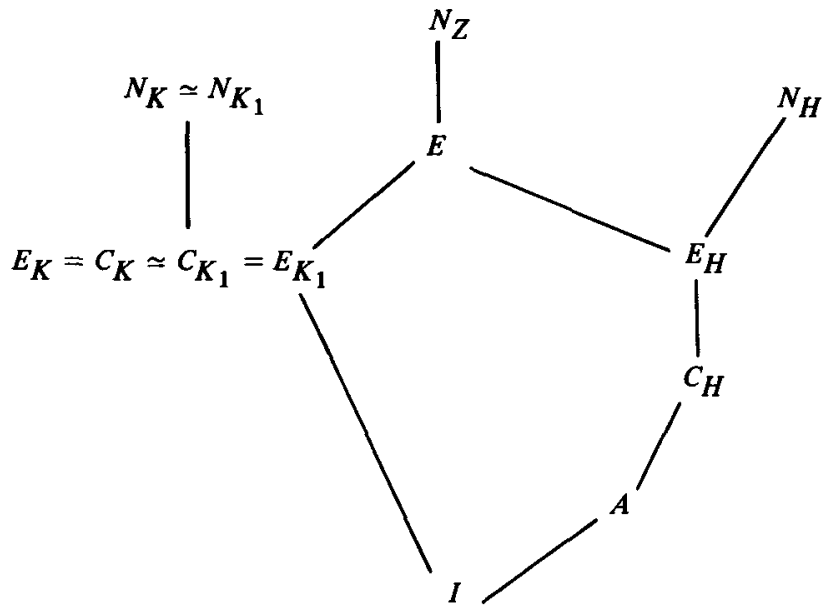


For $G=D_{2 n}, n$ odd, we have already seen that $I=E \subset N_{H}$ for all $H$. In [6] it is shown that $J(E)$ contains an element $\psi$ for which $\psi(a)=a^{w}$. For $H=\langle a\rangle$ then, $\psi \notin M_{0}$ so the inequality of Proposition 3.2 is strict.

Finally let $G=Q_{n}$ be the generalized quaternion group of order $2^{n}, n>3$ with presentation $Q_{n}=\left\langle a, b \mid a^{2^{n}-1}=b a b^{-1} a=a^{2^{n-2}} b^{2}=e\right\rangle$. Then (see [5]) the normal subgroups are precisely the subgroups of $H=\langle a\rangle$, or $K_{1}=\left\langle a^{2}, b\right\rangle$ or $K_{2}$ $=\left\langle a^{2}, a b\right\rangle$ and the automorphisms are of the form $\left[a^{y}, a^{x} b\right]$ for $0 \leqslant x, y \leqslant 2^{\mathrm{n}}$ -1 and $y$ odd. Moreover $|I|=2^{3 n-5}$ and $|A|=|E|=2^{3 n-4}$.

THEOREM 3.6. For $G=Q_{n}, C_{L}=E_{L}=A=E$ for all $L \leqslant H$ and $C_{K_{i}}=E_{K_{i}}=I$.

Proof. Since $I$ has index two in $E$, for every normal $A, C_{A}$ and $E_{A}$ will equal $I$ or $E$. Invoking Proposition 3.2 and looking at the form of the automorphisms we see every automorphism is $L$-continuous for all $L \leqslant H$. On the other hand, only half are $K_{i}$-continuous, namely those $\left[a^{y}, a^{x} b\right]$ with $y$ odd and $x$ even. As shown in [5] the map $(e, e, e, e,--\mid a, a,--, a)$ is in $A$ but it is not $K_{i}$-continuous. The result follows.

\section{Acknowledgments}

The author acknowledges financial support from the NSERC of Canada, and the cooperation of his colleague, Dr. R. D. Small, in producing a computer programme to calculate elements in $E\left(D_{8}\right)$.

\section{References}

[1] Y. Fong and J. D. P. Meldrum, The endomorphism near-rings of the symmetric groups of degree at least five', J. Austral. Math. Soc. Ser. A 30 (1980), 37-49.

[2] P. J. Higgins, An introduction to topological groups (Cambridge University Press, 1974).

[3] R. D. Hofer, 'Near-rings of continuous functions on disconnected groups', J. A ustral Math. Soc. Ser. A 28 (1979), 433-451.

[4] M. Johnson, 'Radicals of endomorphism near-rings', Rocky Mountain J. Math. 3 (1973), 1-7.

[5] J. J. Malone, 'Generalized quaternion groups and distributively generated near-rings', Proc. Edinburgh Math. Soc. 18 (1973), 235-238.

[6] J. J. Malone and C. Lyons, 'Finite dihedral groups and d. g. near-rings I', Compositio Math. 24 (1972), 305-312.

[7] J. J. Malone and C. Lyons, 'Finite dihedral groups and d. g. near-rings II', Compositio Math. 26 (1973), 249-259. 
[8] G. Pilz, Near-rings (North-Holland, Amsterdam, 1977).

[9] E. Schenkman, Group theory (Van Nostrand, Princeton, N. J., 1965).

Department of Mathematics \& Statistics

University of New Brunswick

Fredericton, N. B.

Canada 